\title{
THE EFFECT OF ELECTRICAL OSCILLATIONS ON THE CONDUCTIVITY IMPARTED TO GASES BY AN INCANDESCENT CATHODE.
}

\author{
By ORIN Tugman.
}

HE property of an incandescent carbon filament of emitting electrons in excess of positive electricity has been used by Fleming ${ }^{1}$ for obtaining small direct currents from an impressed oscillatory potential difference of high frequency. Since the emission of negative electricity from the hot carbon tends to discharge a cold positively charged conductor near it a continuous current can be set up through the space between the cold conductor and the hot filament, if the charge on the cold conductor is continually renewed. In Fleming's experiment the carbon filament was surrounded by a metal cylinder as the cold conductor. This cylinder was connected in series with a galvanometer, a coil of wire of few turns and to one terminal of the carbon filament. The coil of wire was electromagnetically coupled to a primary coil in the discharge circuit of a Leyden jar. The induced secondary oscillations can pass in one direction through the vacuous space between the incandescent carbon and the cylinder but not to any great extent in the opposite direction. The direction of the rectified current is, according to the conventional way of stating, from the cylinder to the filament. The oscillations in the opposite direction were almost completely shut out. This device is called by Fleming an oscillation valve.

Lee DeForest ${ }^{2}$ has inserted in the secondary circuit a battery of cells which will of themselves tend to send a current from the cold conductor to the filament. When the oscillations are induced we get the combined effect of the steady applied potential difference and of the oscillatory current. In this device called the "Audion" DeForest has the cold conductor made of metal plates in various

1 Phil. Mag., p. 476. 1905.

${ }_{2}^{2}$ Amer. Institute Elect. Eng. Proc., December, 1906. 
shapes and positions. Recently the U.S. government has issued to DeForest a patent ${ }^{1}$ on a wireless telephone receiver in which there are two metal plates, one on each side of the filament. Marconi $^{2}$ also has a patent device for a similar purpose in which the hot filament is surrounded by a metal cylinder.

The investigation described in the present paper was begun at the suggestion of Professor Merritt, whom the author sincerely thanks for much timely advice throughout the work. The object of the experiment was to determine the currents which could be sent through the space between the cold conductor and the hot filament when the electrical oscillations were superimposed on direct applied potential differences. Also it is intended to show how this current varies with changes in the gas pressure and with the positions of the cold conductor.

The apparatus was set up as shown in Fig. 1. The carbon filaments kindly furnished by the General Electric Co., Harrison, N. $\mathrm{J}$., were mounted at the end of glass tubes one and one quarter inch in diameter. The open end of the tube was fitted to another of the same size attached to an air pump. The joint of the two glass tubes was wrapped with sheet rubber securely wired on. The whole joint was then surrounded by a mercury seal. By this means different filaments could be mounted on the pump in a few minutes. As is shown in the diagram, a glass tubes extends vertically downward from the one holding the filament and ends in a deep cistern of mercury. A long steel rod carrying on its upper end the cold conductor, a brass plate as shown, passes down into the cistern of mercury and turns up on the outside of the tube. This affords a positive connection to the third terminal so its position can be changed at will. Electrical connection is made with the brass plate as shown through the platinum wire sealed in the glass at $c$. A vacuum tube with parallel flat electrodes is sealed on the tube leading to the pump. The discharge of an induction coil through this was used to indicate the pressure of the gas in the apparatus.

The electric oscillations were produced in a coil of eight turns

${ }^{1}$ U. S. Pat. No. $1427,1908$.

2 U. S. Pat. No. 887 , 1908. 
[Vol. XXIX.

and twenty centimeters diameter. One end of this coil was connected through a condenser to earth. The other end of the coil was connected to the third terminal at $c$. The primary coil consisted of three turns twenty centimeters in diameter, connected in series with a Leyden jar and zinc spark gap. The spark gap was

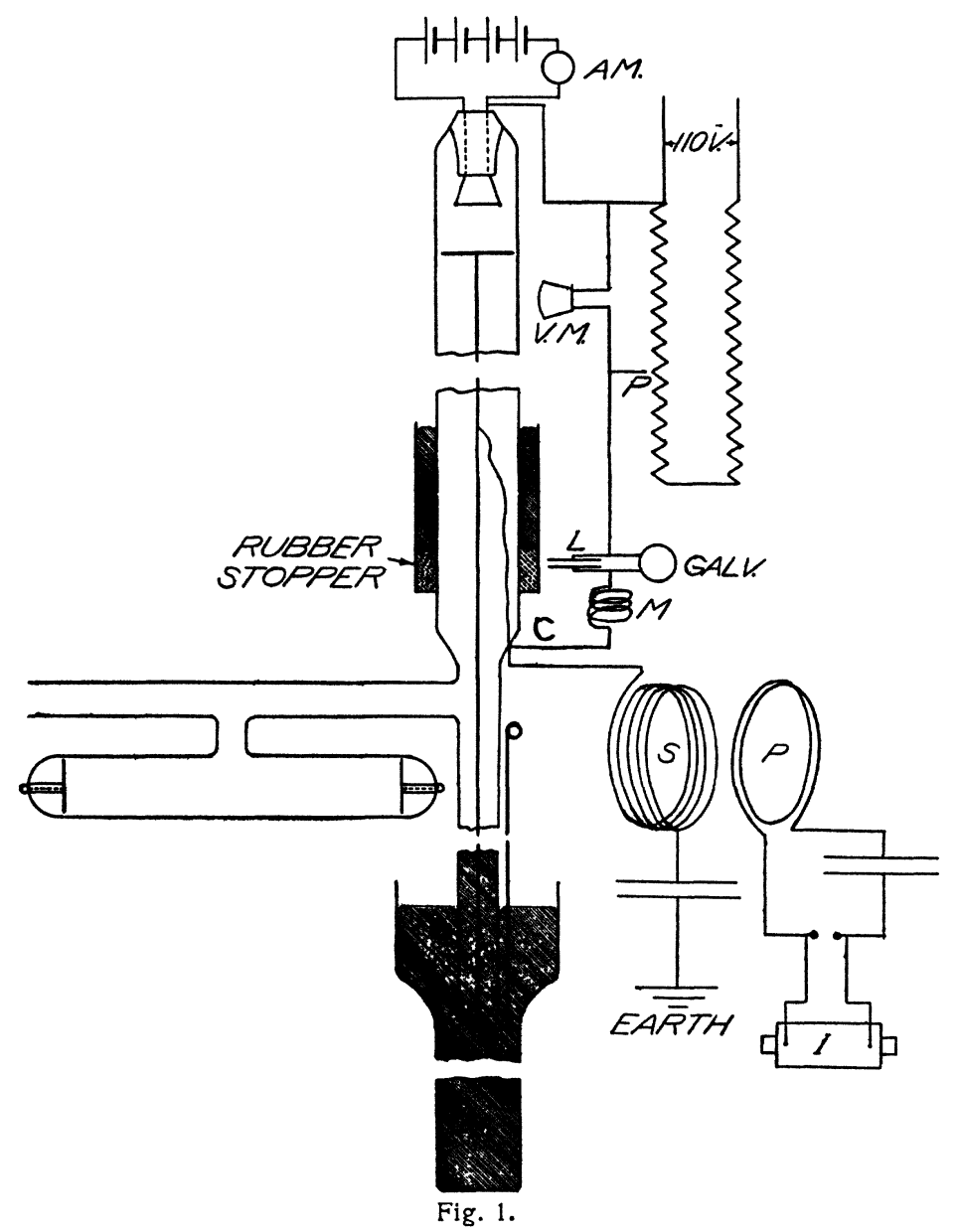

always short so that the oscillations were large in number and of relatively small amplitude, otherwise sparking would occur between the third terminal and the filament. Throughout each series of readings the distance between the two coils and the length of the spark gap remained fixed. 
The direct potential difference could be regulated from the resistance connected to a dynamo circuit and was measured between points $A$ and $B$. Since the currents through the galvanometer are small the fall of potential through it is negligible. The resistance of the galvanometer was $300 \mathrm{ohms}$ and the sensibility $1^{-7}$ amperes per scale division. A variable shunt was used on the galvanometer so as to adapt it for a wide range. To prevent the oscillations from passing through the galvanometer an inductance was placed in series with it at $M$, Fig. I. As a further protection to the galvanometer a Leyden jar was connected in parallel shown at $L$, Fig. I. The carbon filament is heated by an independent storage battery insulated from all other sources of E.M.F.

The first series of observations were made on a horseshoe-shaped filament, burning on from five to seven volts. The third terminal consisted of a brass cup about $2 \mathrm{~cm}$. deep and wide enough to nearly fill the tube. The cup was fixed in one position surrounding the filament and was not changed during this part of the experiment. All the connections were made as shown in the diagram. The air was pumped out until the vacuum gave a cathode ray discharge.

The observations were made in the following manner: The contact $D$ was moved along the resistance $R$ until the desired potential was produced. The current in the galvanometer due to the direct potential difference was then read off the galvanometer scale. After this the oscillations were started by operating the induction coil I. The galvanometer was again read, which reading gave the current through the vacuous space due to the direct potential and the superimposed oscillations.

The results of a series of observations of this kind are shown by the curves in Fig. 2. The abscissæ represent the direct potential difference between the negative end of the filament and the third terminal. The ordinates are the currents through the galvanometer. The continuous lines give the current through the vacuous space when the direct potential is on alone and the broken lines show the current obtained when the oscillations are added to the steady potential. Curves I., II., III., IV. and V. were made when the carbon filament was heated by currents of $2.5,2.7,2.9,3.0$ and 3.1 respectively. 
The general form of the continuous line curves may be explained by considering the manner in which the potential difference between the third terminal and the filament varies as the contact $D$ is moved along the resistance $R$, Fig. I. When the contact $D$ is placed at

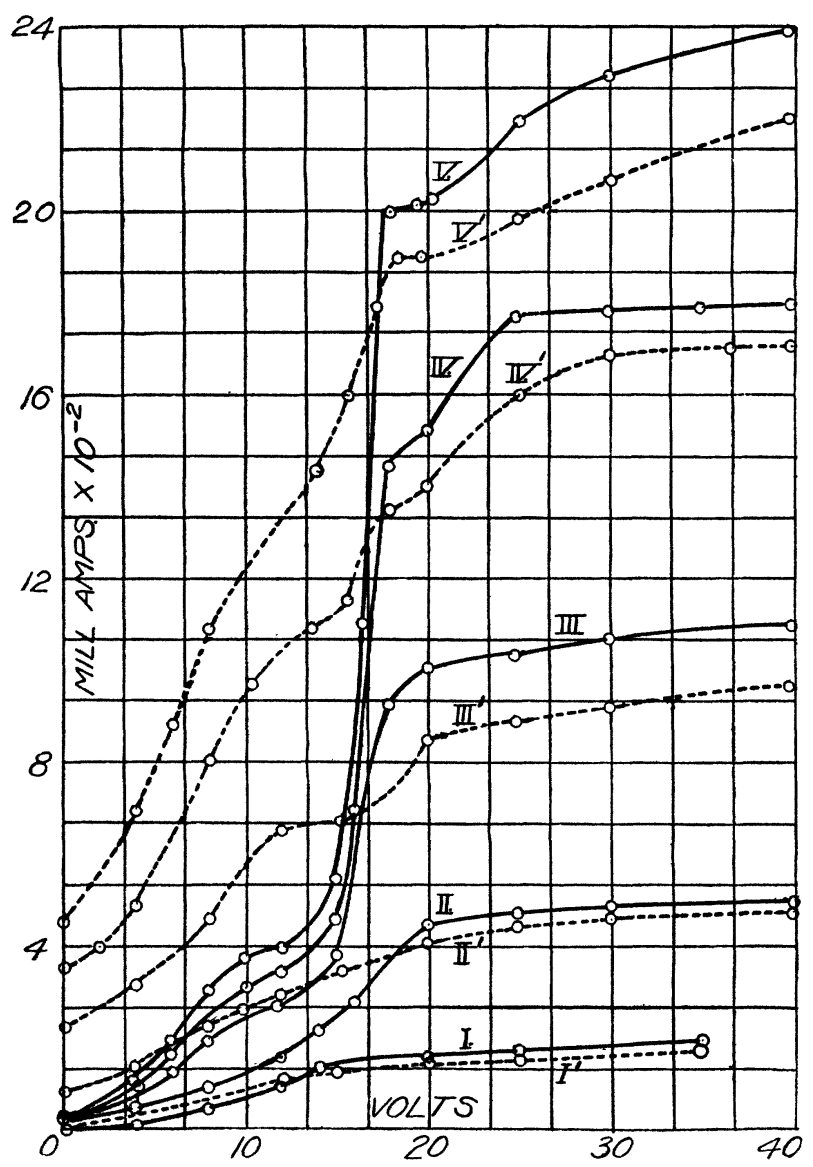

Fig. 2.

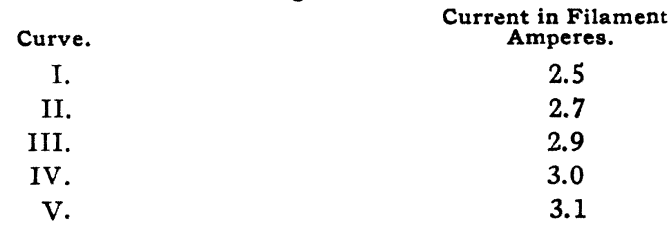

In Curves I.', II.', III.', IV.', V.' the same currents were in the filament as above respectively, but the electrical oscillations were added to the direct potential difference. 
$E$ the third terminal has the same potential as the negative end of the filament. The galvanometer showed practically no current with this connection. If the contact $D$ is moved a short distance along $R$ the potential of the third terminal will be equal to the potential of some point on the filament, a short distance from the

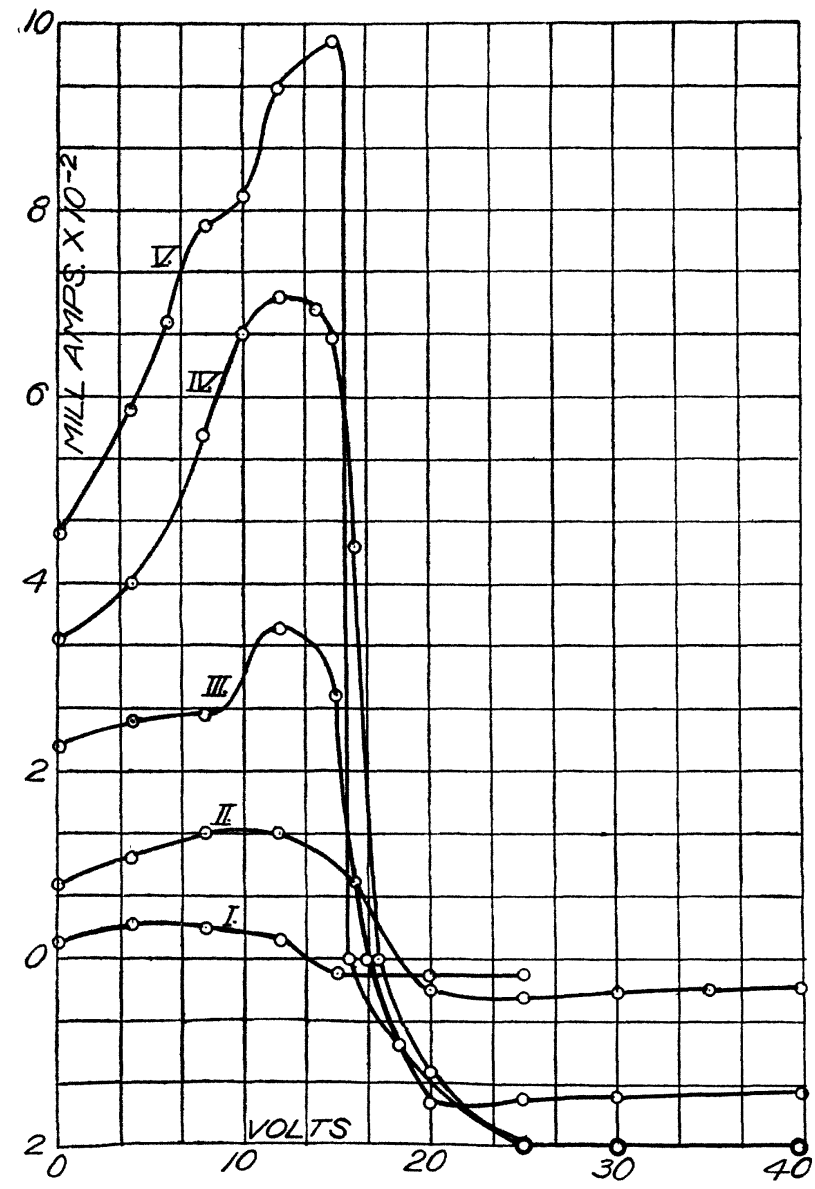

Fig. 3.

Curve.

I.

Current in Filament

II.

Amperes.

III.

2.5

2.7

2.9

IV.

3.0

v.

3.1 
negative end. Consequently electrons will now pass from the negative end of the filament to the third terminal. As higher potential differences are applied the third terminal will receive electrons from a greater portion of the filament until all the electrons emitted by the hot carbon reach the third terminal. When this state is reached the number of elections emitted by the hot carbon is not greatly influenced by any further increase in the potential difference between the third terminal and the filament. We see from the curves in Fig. 2 that the current through the galvanometer is saturated at about fifteen volts. The continuous line curves are similar to those obtained by Merritt and Stewart ${ }^{1}$ with a metal cylinder surrounding a carbon filament.

The broken line curves in Fig. 2 show the results obtained by superimposing the electrical oscillation on the direct potential difference. It is seen from the curves that the electrical oscillations produce a current through the galvanometer even when the third terminal is at the same potential as the negative end of the filament. This is to be expected when we consider that only one half of the wave can pass through the vacuous space. When the third terminal is charged negatively by the oscillations the electrons are repelled and cannot take part in the conduction of electricity. Furthermore, since the number of positively charged particles emitted by hot carbon is so very small compared to the number of electrons, the negative half of the oscillation is cut out and current passes through the galvanometer only when the oscillations charge the third terminal positively. The potential produced by the oscillations is probably more than great enough to produce saturation. But this potential lasts for a short time and the duration of each train of oscillations is very small compared to the interval between each train of oscillations. Therefore it seems reasonable that the galvanometer current should not be saturated when the high oscillatory potential difference is superimposed on a small direct potential difference. The same line of reasoning will explain the decrease of the galvanometer current, produced by the oscillations, after saturation. When the direct potential difference has produced the saturation current, any farther increase in the potential difference

' Phys. Rev., p. 239, 1904. 
cannot increase the number of electrons emitted by the hot carbon. But more that half of the oscillation wave which charges the third terminal negatively becomes effective in reducing the difference in potential between filament and third terminal. As a consequence of this reduced potential difference the galvanometer current falls below the saturation value.

By plotting the differences in the currents obtained by the direct potentials alone and that obtained by the resultant of the direct and oscillatory potentials we have the curves shown in Fig. 3. After

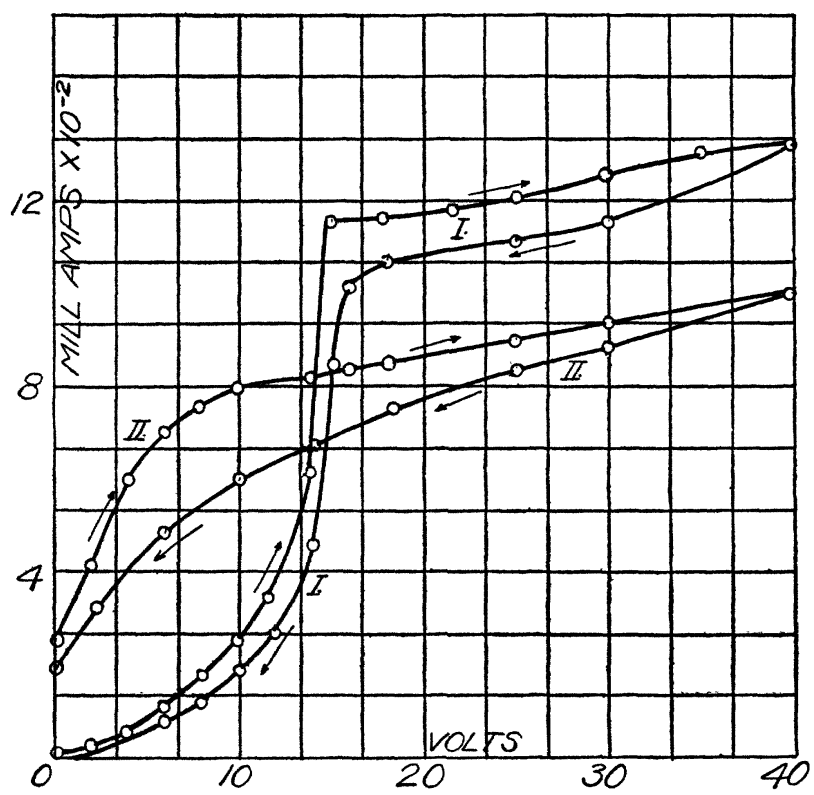

Fig. 4. Current in Filament, 3 amps.

reaching forty volts and the observations were made coming down the curve, it was found that the descending curve had shorter ordinates than the ascending curve. Such a curve is shown in Fig. 4. It is seen that the galvanometer current is greater with increasing voltages than with decreasing potential differences.

In all the above experiments the brass cup, as a third terminal, remained fixed in one position. It was thought that the position of the third terminal would have an interesting effect on the galvanometer current. In the remaining experiments the device previously 
described and shown in the diagram (Fig. I) was used to vary the position of the third terminal. In this part of the investigation the third terminal was a brass plate three centimeters in diameter, mounted as shown in the diagram. The first experiment was made

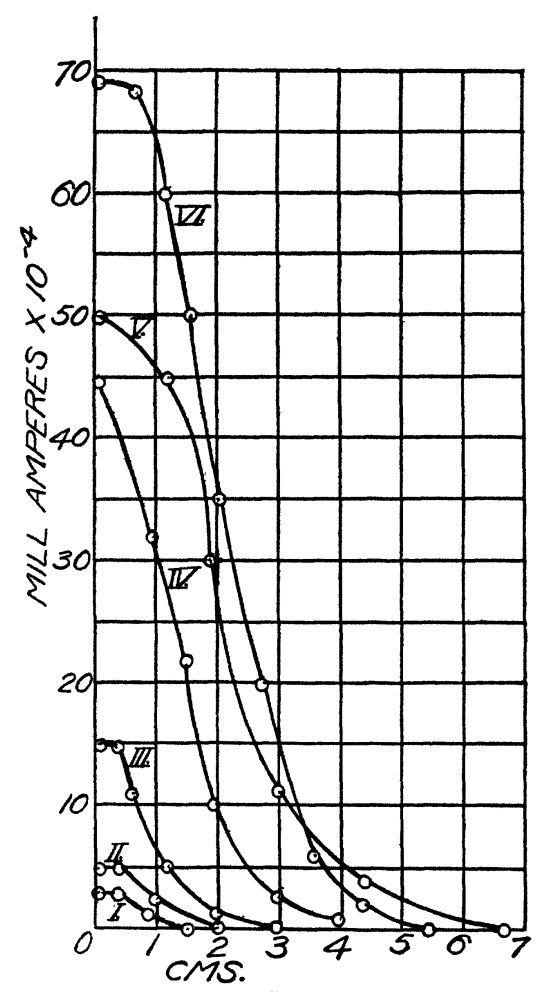

Fig. 5. Current in lamp filament for Curves I., II., III., IV., V. I.6 amp. for Curves VI. and VIII.

$$
\begin{gathered}
\text { Curve. } \\
\text { I. } \\
\text { II. } \\
\text { III. } \\
\text { IV. } \\
\text { V. } \\
\text { VI. }
\end{gathered}
$$$$
\text { Potential Difference between Filament }
$$$$
\text { and Third Terminal Volts. }
$$
0
100

25

50

75

100

with a horse-shoe-shaped filament carrying 1.5 amperes on 60 volts. The brass plate was so adjusted that its distance from the bottom of the carbon loop was about half of a millimeter. The results of some experiments made without the electrical oscillations, but with a direct 
applied potential difference, are shown in Fig. 5. Curves I., II., III., IV. and V. were made when $0,25,50,75$ and 100 volts direct were impressed respectively, and in all five the current in the filament was I.6 amperes. In Curve VI. the current in the filament was 1.8 amperes and the potential applied to the third terminal was IOO volts.

It is to be seen that in most of these curves the current in the galvanometer does not drop off quickly as the brass plate is removed until nearly half a centimeter intervenes. All the distances plotted along the $x$-axis were reckoned from the lowest point of the curved

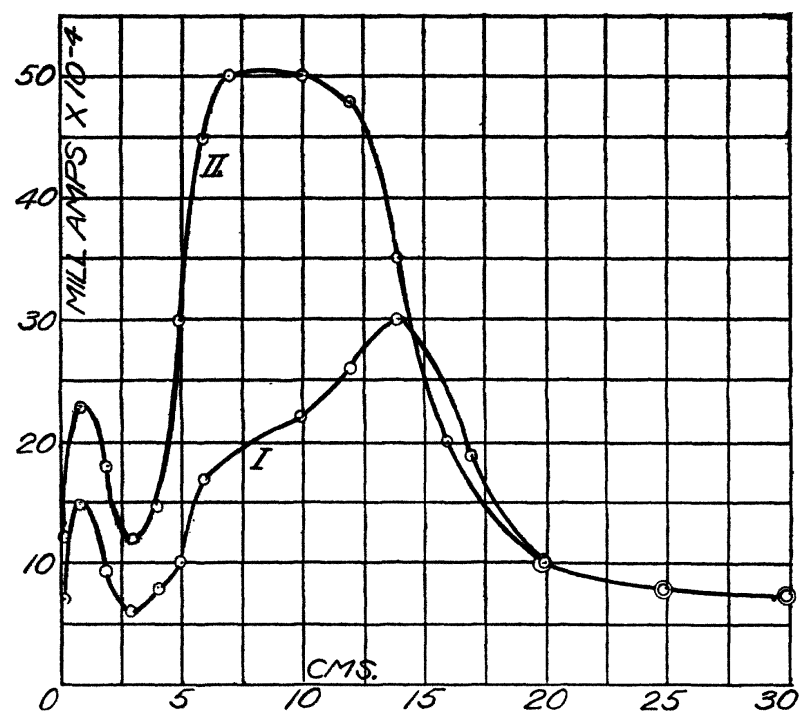

Fig. 6. I.2 amp. in lamp filament; I., zero potential difference; II., 25 volts. potential difference.

filament which hung in a vertical plane with its convex side down. Also the vacuum was high enough for the vacuum tube to give a good cathode ray discharge.

More interesting results are obtained when a straight carbon filament is used in place of the curved one. This permits the brass plate to be the same distance from all parts of the carbon. Adjustments were made so that the brass plate could be brought up very close to the incandescent filament. Some results are shown in Figs. 6,7 and 8. As the brass plate is moved away from the fila- 
[Vol. XXIX.

ment the current through the galvanometer at first increases with the distances. When the brass plate is nearly a centimeter off the galvanometer current is in some cases nearly twice as large as when the plate is a millimeter distant from the filament. Figs. 6 and 7 also show a decided increase in the galvanometer current when the plate is out about five centimeters. All of these curves were made with the oscillations on. The pressure was not as low as could be produced, the cathode of the discharge tube showing a large blue spot. After pumping for some time and repeating the observations curves like those in Fig. 8 were obtained. Here we have a maximum very pronounced at about one centimeter. After this point is passed the curve falls off rapidly at first and then gradually slopes

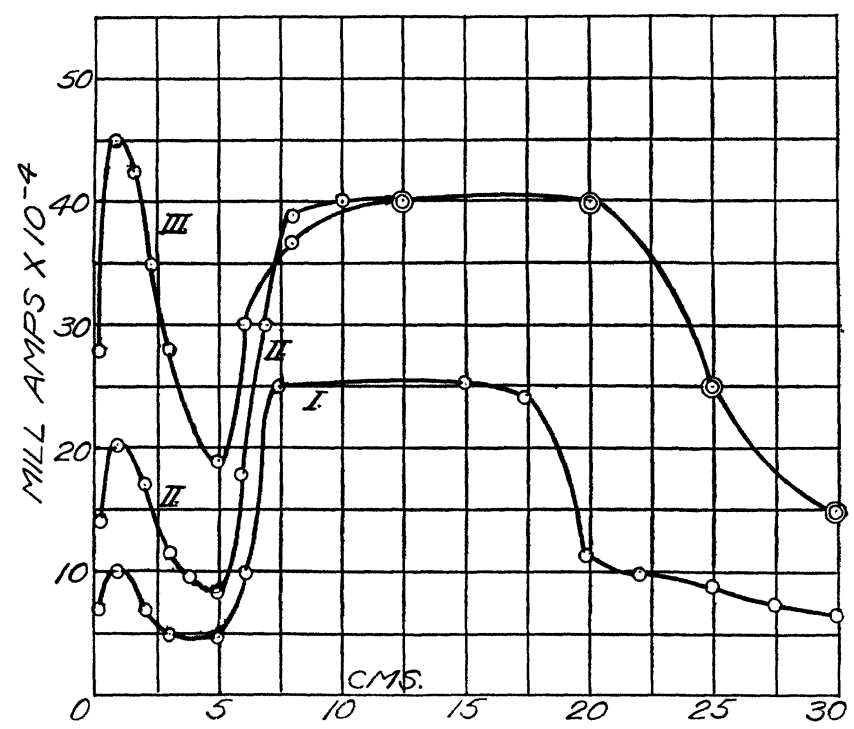

Fig. 7. I amp. in lamp filament; I., zero potential difference; II., 25 volts potential difference; III., 50 volts potential difference.

off to zero. Continuous pumping was necessary to keep the second maximum from appearing. We see in Fig. 8, curve III. shows a slight tendency to increase after the first rapid decrease. From this it seems that the pressure of the gas determines the presence of the second maximum. Also it is noted that as the pressure is lowered the first maximum gets more pronounced. 
A series of observations was made using only the direct potential. A most decided maximum in some of these curves appears when the third terminal is about one centimeter from the filarnent. These are shown in Fig. 9. When the higher voltages are applied the per cent. of increase of current from the beginning to the highest

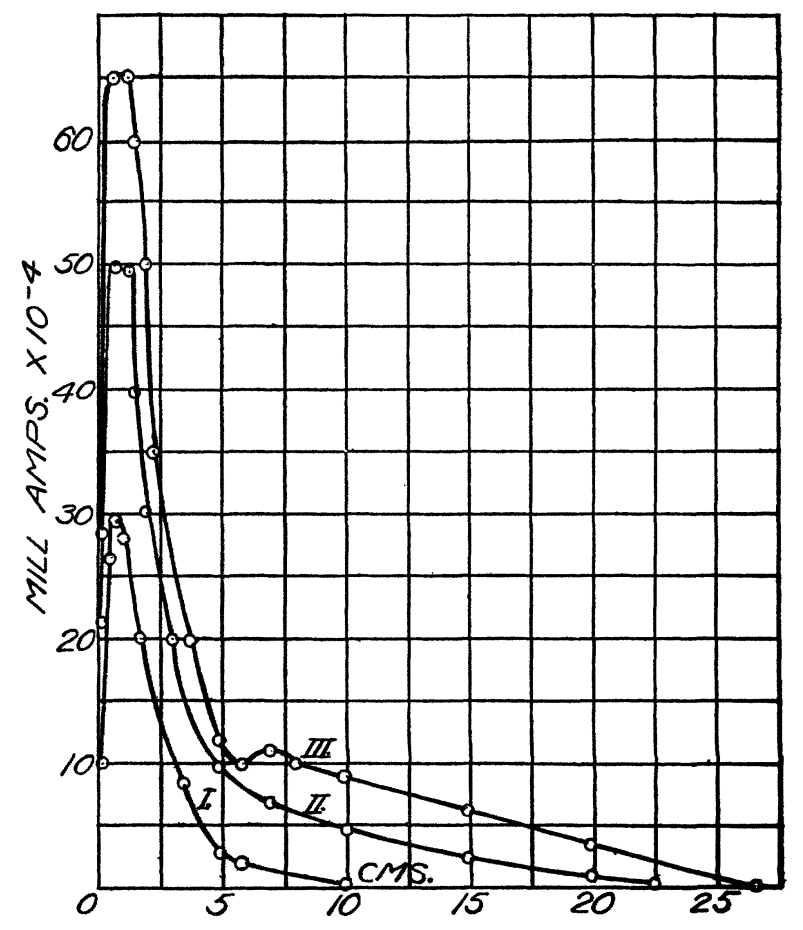

Fig. 8. I.3 amp. in lamp filament; I., 20 volts potential difference; II., 30 volts potential difference; III., 40 volts potential difference.

value is greater than for the low voltages. Fleming made some experiments in which he measured the current obtained from the negative leg of a carbon loop to a metal plate. His curves correspond to those in Fig. 9 from the maximum point down.

If the filament current and the direct potential are maintained constant and the pressure varied some more data are given, which show under what conditions the second maximum appears when the oscillations are on. In Fig. Io, curve I. was made when the vacuum tube discharge was gray in color, just before cathode rays 
appear. Curve II. was made just as the first faint traces of cathode rays appeared. For curve III. the vacuum tube showed full cathode ray discharge, the blue spot on the cathode being a small blue point. Curves IV. and V. show a similar change as the air is pumped out. It can be seen here that the first maximum is increased with diminished pressure.

A reasonable explanation of the first maximum may be obtained from an analogy to the discharge through vacuum tubes. It is known ${ }^{1}$ that the effect of cathode ray particle by impact on the gas

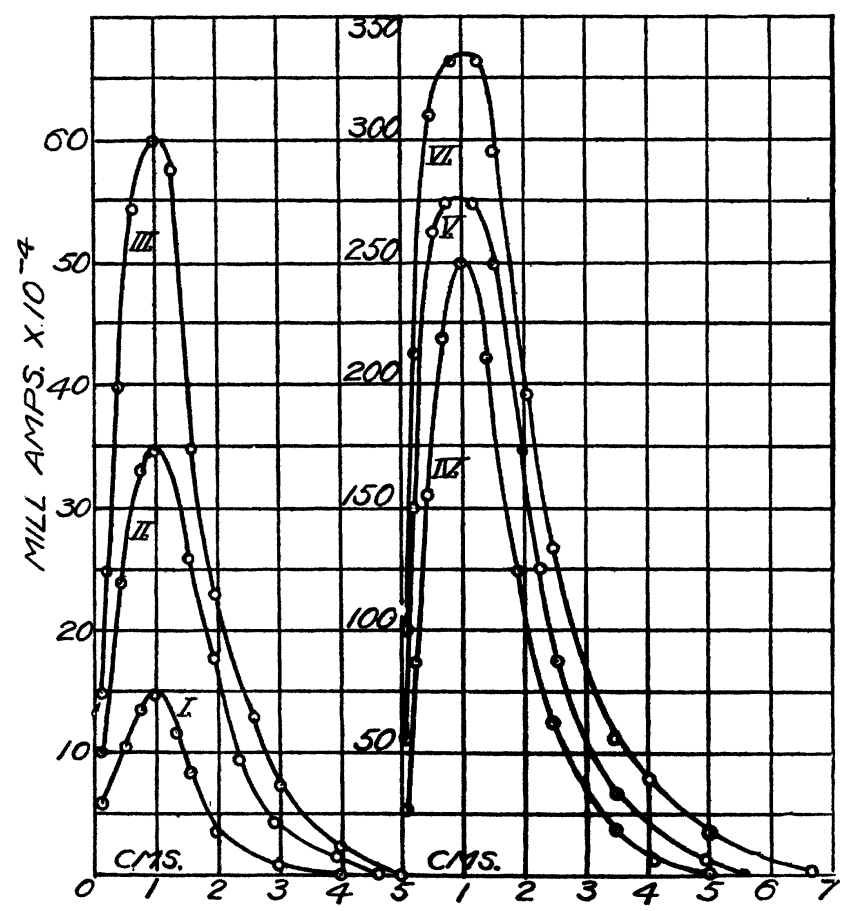

Fig. 9. I.2 amps. in lamp filament; I., 40 volts potential difference without oscillations; II., 50 volts potential difference; III., 60 volts potential difference; IV., 70 volts potential difference; V., 80 volts potential difference; VI., 90 volts potential difference.

molecules is small close to the cathode. The ionizing effect of the cathode rays increases rapidly as the negative glow is approached, reaches a maximum in the glow and quickly drops to a small

$1 \mathrm{~J}$. J. Thompson, Conduction of Electricity through Gases, p. 484. 
value. In the present case the brass plate in the nearest position to the hot filament is within the cathode dark space. As the third terminal is moved away from the filament regions are reached where

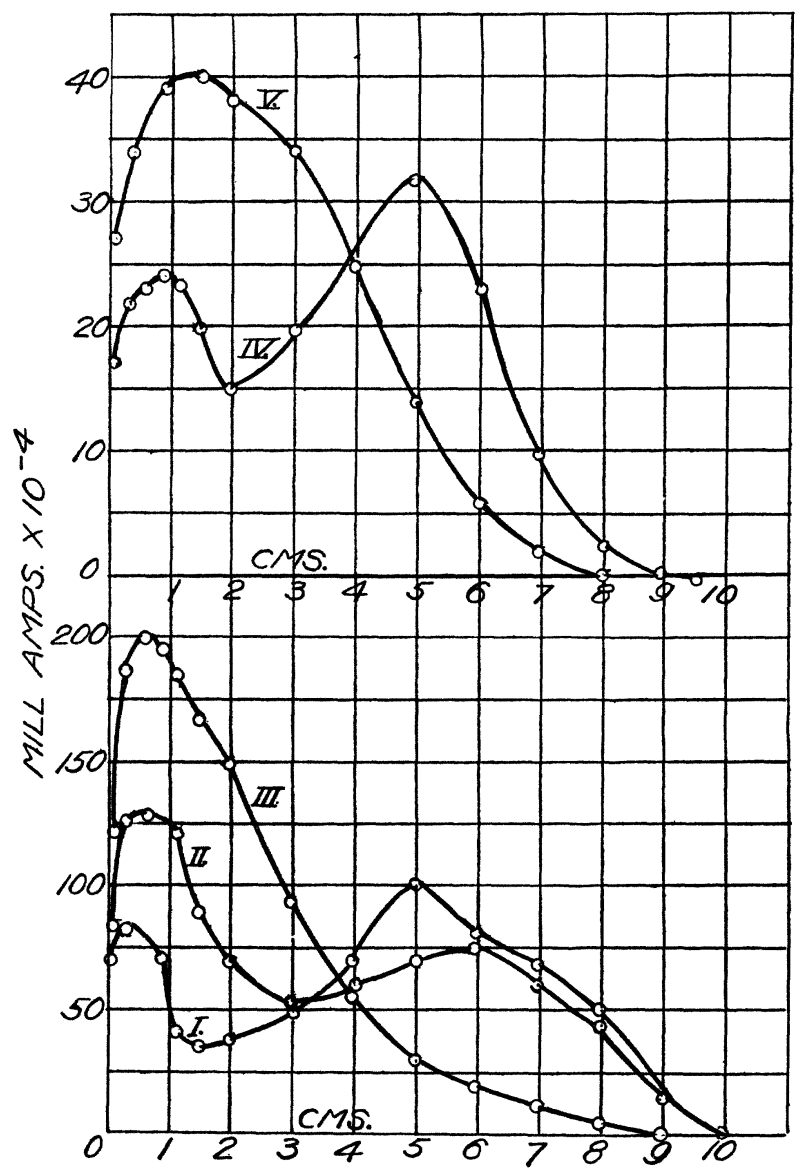

Fig. 10. I., II., III., current in lamp filament, 1.4 amp., 23 volts ; IV., V., current in lamp filament 1.2 amp., 30 volts.

the electrons from the carbon have greater ionizing power. After passing this point the galvanometer current falls off.

The second maximum must be due to the secondary ionization in the gas. As was cited above, this second maximum occurred only at relatively high pressures, $i . e$., pressures greater than that required for good cathode rays. Furthermore, when the ampli- 
[Vol. XXIX.

tude of the oscillation was small, the potential gradient was not sufficient to give the ions the required velocity for ionization.

In Fig. I I are shown some curves made when the direct potential was comparatively high. It will be remembered that in Fig. 2

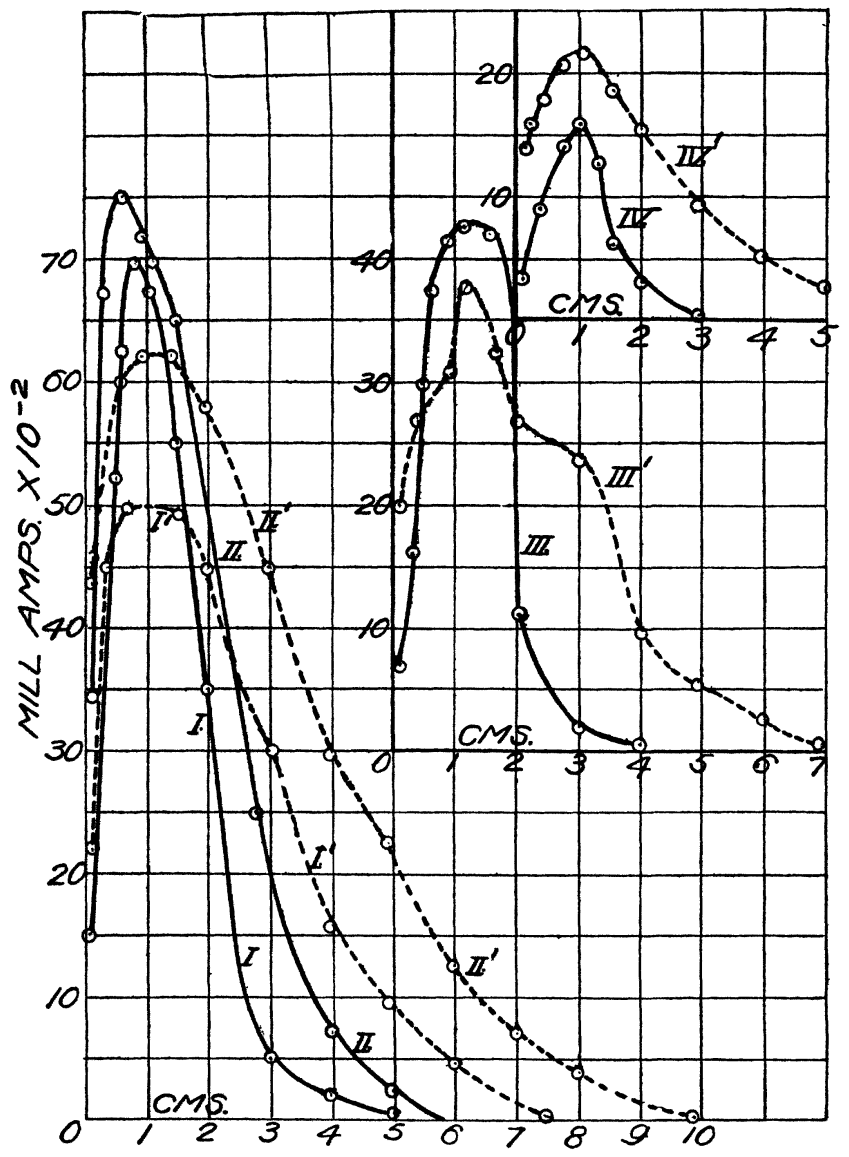

Fig. 11. 8 amp. in lamp filament; I., 50 volts without oscillations; I.', 50,volts with oscillations; II., 60 volts without oscillations ; II.', 60 volts with oscillations ; III., 70 volts without oscillations ; III.' 7 O volts with oscillations ; IV., 80 volts without oscillations ; IV.', 80 volts with oscillations.

the galvanometer current is decreased by the oscillations when saturation exists. In Fig. I I we see that the oscillations produce a decrease in the galvanometer deflections when the brass plate is about one centimeter distant. After this distance is passed there is 
an increase of the galvanometer current due to the oscillations. The continuous line as before represents the current produced by the direct potential alone and the broken line when the oscillations are impressed on the direct potential. In all these curves the same current flowed in the filament and the pressure was low enough to prevent the second maximum from appearing. In curves $V, V^{\prime}$, the direct potential is 40 volts. Under this condition the effect of the oscillation is always to increase the current through the gas. The highest maximum on this curve sheet is reached when about 80 volts is applied.

It appears that different filaments have not acted the same with regard to the potential required to produce saturation. In these last curves apparently over 50 volts must be used, whereas in Fig. 2 saturation is produced at about 15 volts.

\section{Summary.}

When the direct potential between the third terminal and the hot carbon is not producing the saturation current, the effect of the electrical oscillations is to increase this current and to decrease it if the saturation is being already produced.

The distance between the third terminal has a marked effect on the current through the vacuous space. In these experiments the current reaches a maximum when the third terminal is about one centimeter distant from the filament. A second maximum occurs when the gas pressure is not too low and the oscillating potential high enough to produce ionization.

As the age of the filament increases with use the sensitiveness of all these effects decreases.

Physics Laboratory,

CORNELL Universit'y. 


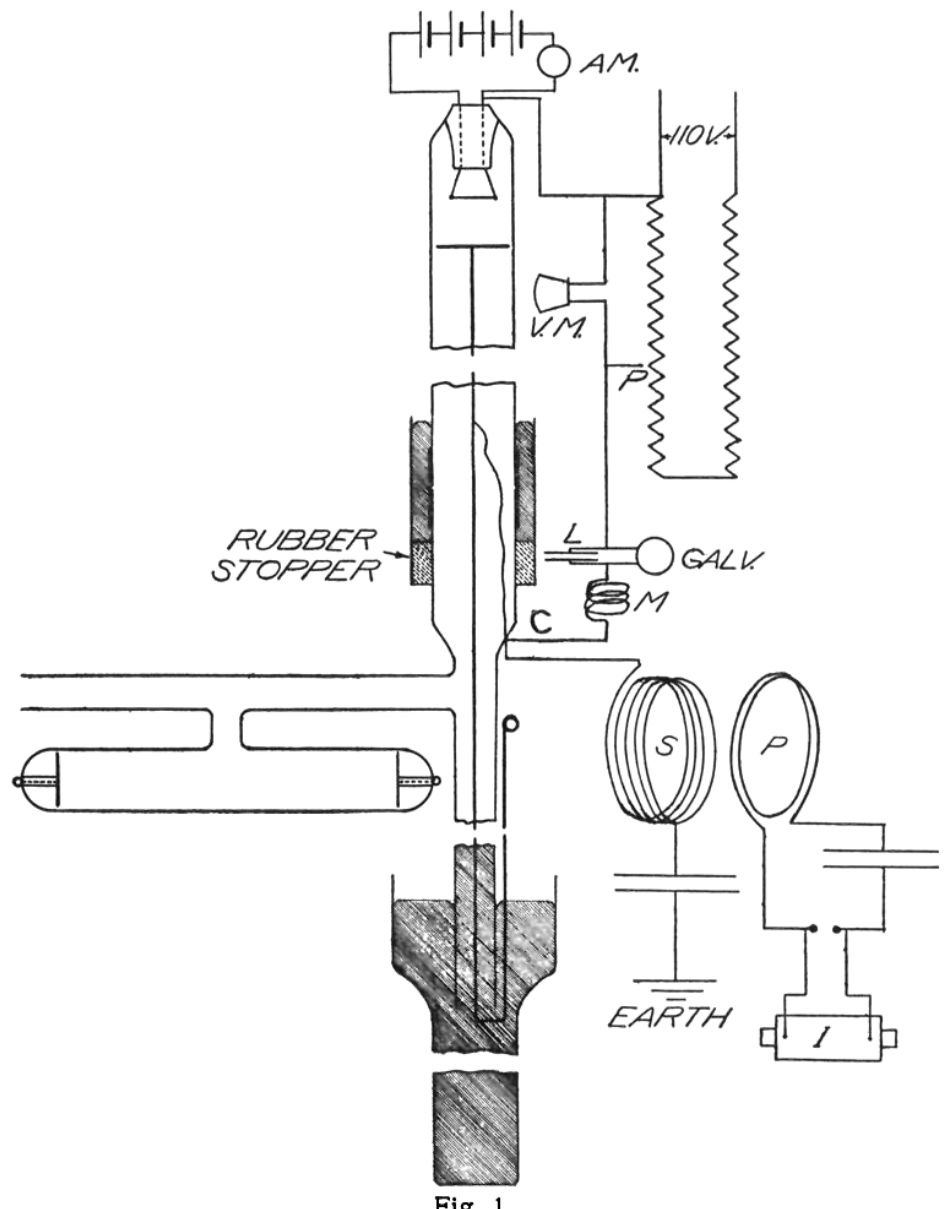

Fig. 1. 